\title{
Parametrization of nonlinear and chaotic oscillations in driven beam-plasma diodes
}

\author{
Min Sup Hur, Hae June Lee, and Jae Koo Lee \\ Department of Physics, Pohang University of Science and Technology, Pohang 790-784, South Korea
}

(Received 20 January 1998)

\begin{abstract}
Nonlinear phenomena in a driven plasma diode are studied using a fluid code and the particle-in-cell simulation code XPDP1. When a uniform electron beam is injected to a bounded diode filled with uniform ion background, the beam is destabilized by the Pierce instability and a perturbation grows to exhibit nonlinear oscillations including chaos. Two standard routes to chaos, period doubling and quasiperiodicity, are observed. Mode lockings of various winding numbers are observed in an ac driven system. A new diagnostic quantity is used to parametrize various nonlinear oscillations. [S1063-651X(98)00707-7]
\end{abstract}

PACS number(s): 52.35.Fp, 52.35.Qz, 52.40.Mj

\section{INTRODUCTION}

Self-oscillations and standard routes to chaos were observed in many experiments or numerical simulations of plasma [1-15] and free-electron laser systems [16-20]. It is important to represent such oscillations in the parameter windows for further studies and design of similar systems. In this work, we focus on the parametrizing of a beam plasma model-the Pierce-type plasma diode $[1,15]$ with an external driver. This plasma diode is a bounded collisionless system with an electron beam in a uniform ion background. One electrode (cathode) is grounded, where an initially uniform electron beam is injected. The other electrode (anode) is driven by $\mathrm{dc}$ and ac voltages. The ions are fixed, forming a uniform background with nearly the same charge density as the injected electron beam.

Generally, there are many input parameters that can control the system states: system length $L$, the initial velocity of the electron beam $u_{0}$, the ratio of ion density to initial electron density $\eta=n_{i} / n_{0}$, the dc driving voltage $V_{\mathrm{dc}}$, the amplitude $V_{\mathrm{ac}}$, and the frequency $\omega_{e}$ of the ac driving voltage. It is known that $L$ and $u_{0}$ can be combined to one main parameter called the Pierce parameter $\alpha=\omega_{p} L / u_{0}$ [1] where $\omega_{p}$ is the electron plasma frequency. The effect of $\eta$ was studied by Chen and Linsay [21]. The effects of the driving voltage and the frequency on the beam plasma systems were investigated in many simulations and experiments [3-14]. Because of many control parameters, the parameter windows usually show complex structures. We use a new diagnostic parameter, with which such complex structures can be reduced to simple diagrams. This diagnostic parameter originated from our previous FEL simulations [16-18] is defined in a similar way for the plasma diode system [22].

In Sec. II the fluid equations describing the system are presented. In Sec. III we present the results of simulations for the dc driven cases, the ac and dc driven cases, and the input parameter windows. In Sec. IV the system state is described using a parameter that is suggested as a universal diagnostic quantity for the beam-plasma systems. The conclusion of this paper is summarized in Sec. V.

\section{SIMULATION}

Simulations were done with a fluid code [22] and the kinetic code XPDP1 [23]. The diode is described by following one-dimensional fluid equations with proper boundary conditions:

$$
\begin{aligned}
& \frac{\partial n}{\partial t}+\frac{\partial}{\partial x}(n u)=0, \\
& \frac{\partial u}{\partial t}+u \frac{\partial u}{\partial x}=\frac{e}{m} \frac{\partial V}{\partial x}, \\
& \frac{\partial^{2} V}{\partial x^{2}}=\frac{e}{\epsilon_{0}}\left(n-n_{i}\right),
\end{aligned}
$$

where $V$ is the plasma potential, $n$ is the electron number density, $n_{i}$ is the background ion density, $u$ is the velocity of the electron stream, $m$ is the electron mass, and $\epsilon_{0}$ is the permittivity of free space. Because the ions are fixed, there is no dynamic equation about ion motion. Ionization is assumed to be negligible; this effect is the subject of our future study. We define the coordinates $x=0$ as the cathode location and $x=L$ as the anode location. The boundary conditions become

$$
\begin{aligned}
V(0, t)=0, \quad V(L, t) & =V_{\mathrm{dc}}+V_{\mathrm{ac}} \sin \left(\omega_{e} t\right), \\
u(0, t) & =u_{0}, \\
n(0, t) & =n_{0} .
\end{aligned}
$$

The variables in these equations can be properly normalized to reduce the equations into simple forms:

$$
\begin{aligned}
& \frac{\partial N}{\partial T}+\frac{\partial}{\partial X}(N U)=0, \\
& \frac{\partial U}{\partial T}+U \frac{\partial U}{\partial X}=-\frac{\partial \phi}{\partial X}, \\
& \frac{\partial^{2} \phi}{\partial X^{2}}=-\alpha^{2}(N-\eta),
\end{aligned}
$$

with

$$
\begin{gathered}
N=\frac{n}{n_{0}}, \quad T=\frac{v_{0} t}{L}, \quad X=\frac{x}{L}, \quad U=\frac{u}{u_{0}}, \quad \eta=\frac{n_{i}}{n_{0}}, \\
\phi=-\frac{e V}{m u_{0}^{2}}, \quad \alpha=\frac{\omega_{p} L}{u_{0}}, \quad \omega_{p}=\sqrt{\frac{e^{2} n_{0}}{m \epsilon_{0}}}
\end{gathered}
$$


The Pierce parameter is a significant control parameter. The fluid code used here is based on these normalized equations and all the simulation results are represented in the dimensionless parameter space of $\alpha, \phi_{\mathrm{dc}}$, and $\phi_{\mathrm{ac}}$ instead of $u_{0}, L$, $V_{\text {dc }}$, and $V_{\text {ac }}$. The kinetic code does not use these fluid equations but the same normalization convention was used to compare the results from two different codes.

\section{RESULTS}

The normalized input parameters in Eq. (10) are used in the fluid simulations. In the kinetic simulations, all the input parameters are specified in SI unit. The initial velocity and density are chosen as $u_{0}=2 \times 10^{6} \mathrm{~m} / \mathrm{s}$ and $n_{0}=10^{12} \mathrm{~m}^{-3}$, respectively. In this regime, the electron plasma frequency is $\omega_{p}=5.6 \times 10^{7}$. To change $\alpha$, the system length $L$ is varied with fixed $u_{0}$. $L$ is about $0.3 \mathrm{~m}$. Some cases with a different $u_{0}$ but the same $\alpha$ yielded the same results, meaning that the dimensionless value $\alpha$ is more important than each of $u_{0}$ and $n_{0}$ even in the kinetic simulations.

It is well known that a dissipative system undergoes period doublings, quasiperiodicities, or intermittencies to reach chaos. In this work, the first two routes were found to be dominant.

\section{A. dc driven cases}

When the system is driven by a dc voltage with $V_{\mathrm{ac}}=0$, it becomes chaotic through the period doublings as $V_{\mathrm{dc}}$ is increased for a fixed $\alpha$ or as $\alpha$ is decreased for a fixed $V_{\mathrm{dc}}$. Figure 1 shows the time evolutions of the electric field at the cathode and their power spectra calculated from the kinetic code. The Pierce parameter is fixed as $\alpha / \pi=2.98$ and the normalized voltage is varied from $\phi_{\mathrm{dc}}=0$ to 0.18 . A clear period-doubling route is found: stable focus for $\phi_{\mathrm{dc}}<0.03$, self-oscillation of period one for $0.03<\phi_{\mathrm{dc}}<0.11$, period two and four for $0.11<\phi_{\mathrm{dc}}<0.14$, chaotic oscillation for $0.14<\phi_{\mathrm{dc}}<0.17$, and unstable state for $0.17<\phi_{\mathrm{dc}}$. The unstable state is defined as follows: when the system is in a stable focus state or an oscillating state, the velocities of all electrons in the beam are positive, i.e., with electrons moving from cathode to anode. In some parameter regimes, the electrons start to be reflected near the electrodes due to the virtual cathode. Then the stream becomes turbulent before going to the limited current state. This unstable regime can be calculated by the equilibrium analysis [24,25].

There are strong correlations between the linear growth rates and the saturated states. In Fig. 2, the solid lines are the linear growth rates and the dashed lines are the linear frequencies calculated from the linearized fluid equations (7)(9) with various $\eta$ and $\phi_{\mathrm{dc}}$. The system tends to be more chaotic when the linear growth rate is large. The nonlinear growth rates and frequencies are obtained from fluid equations (7)-(9). The nonlinear frequencies determine the phase velocities of the wave, which are important factors in beamplasma interactions as described in Sec. IV.

From fluid simulations, the Feigenbaum constant $\delta$ [26] for period doubling up to $16 P$ was calculated in terms of $\alpha$ [22]. The $\delta$ was 4.8308 and 4.6429 for $\phi_{\mathrm{dc}}=0, \eta=1 ; 4.7586$ and 4.8333 for $\phi_{\mathrm{dc}}=0.05, \eta=1 ; 4.9714$ and 4.6667 for

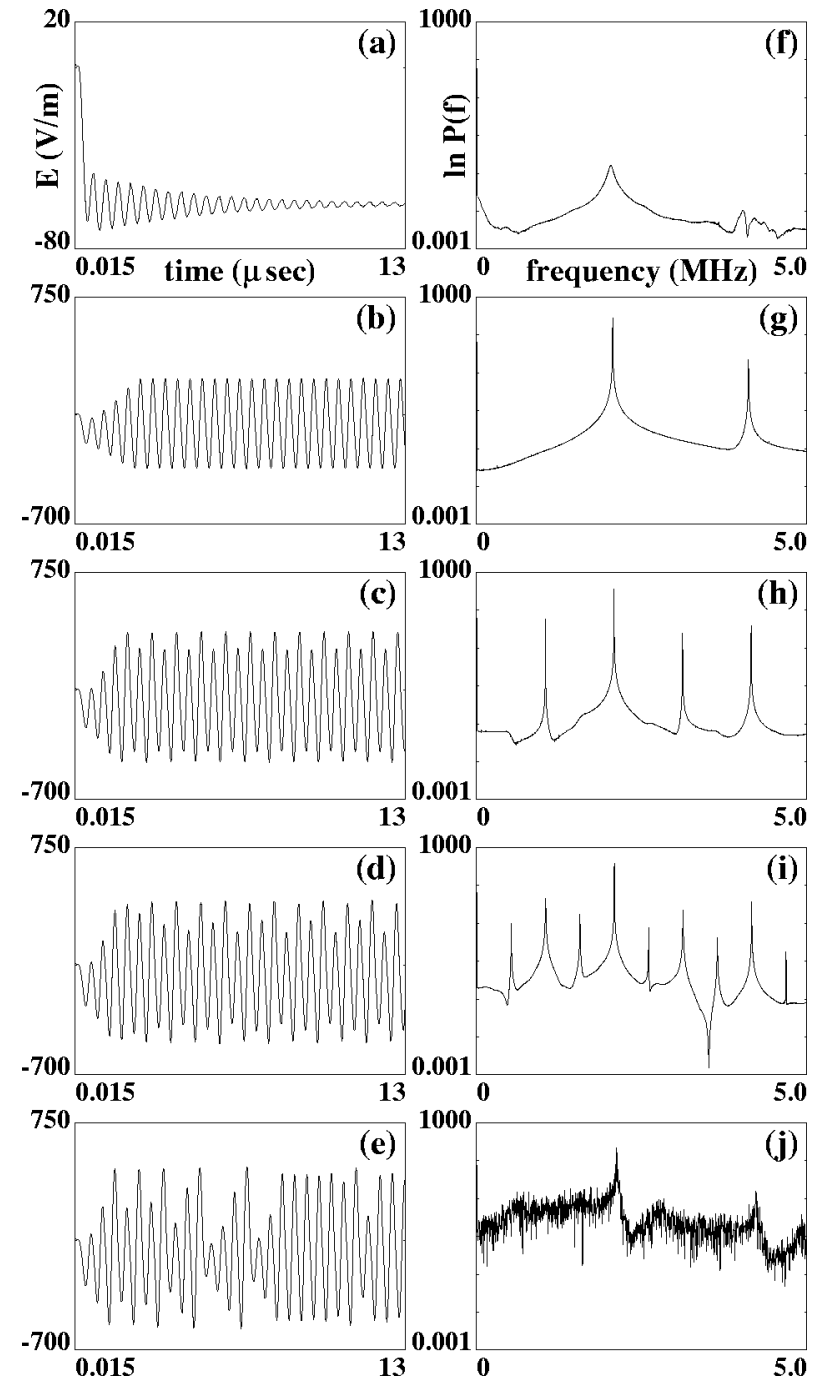

FIG. 1. A period-doubling route to chaos with $\alpha / \pi=2.98$ from kinetic simulations. (a) Stable focus, $\phi_{\mathrm{dc}}=0.02$. (b) $1 P, \phi_{\mathrm{dc}}$ $=0.08$. (c) $2 P, \phi_{\mathrm{dc}}=0.126$. (d) $4 P, \phi_{\mathrm{dc}}=0.134$. (e) Chaos, $\phi_{\mathrm{dc}}$ $=0.16$. $(\mathrm{f}),(\mathrm{g}),(\mathrm{h}),(\mathrm{i}),(\mathrm{j})$ are the corresponding power spectra.

$\phi_{\mathrm{dc}}=0, \eta=1.05$. These values are close to the universal value 4.6692 .

\section{B. ac and de driven cases}

If an ac voltage is added to the driving force with amplitude less than the dc voltage, two main frequencies coexist to make a quasiperiodicity (denoted as QP). One of the main frequencies is the self-oscillation frequency and the other is the frequency of the external ac voltage. Figure 3(b) is the power spectrum of QP. There are two main peaks: the external frequency $f_{1}=1.2 \times 10^{6} \mathrm{~Hz}$ and the self-oscillation frequency $f_{2} \simeq 2 \times 10^{6} \mathrm{~Hz}$. Most of the small peaks are represented as the combinations of $f_{1}$ and $f_{2}$ with integral coefficients [27]. Figure 3(f) is the return map of QP. The horizontal axis represents $E_{0}(t)$ (the electric field at the cathode at time $t$ ) and the vertical axis represents $E_{0}(t+T)$ (the electric field at time $t+T)$. Here $T$ is the period of driving ac voltage, so this return map corresponds to the Poincaré section of a two-dimensional torus [27]. Therefore the shape of the return map should be topologically similar to a circle. As the system is driven by $V_{\mathrm{dc}}$ more strongly with the existence of $V_{\text {ac }}$ rather small compared to the $V_{\mathrm{dc}}$, the power spectrum 


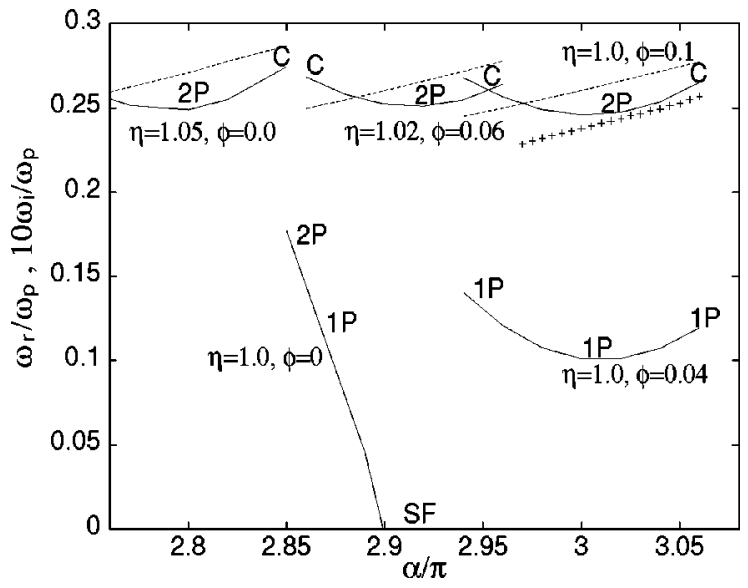

FIG. 2. Growth rates and system states for various $\alpha, \phi_{\mathrm{dc}}$, and $\eta$ from fluid simulations. The vertical axis represents the real frequency $\omega_{r}$ and the growth rate $\omega_{i}$ (multiplied by 10) normalized with respect to the electron plasma frequency $\omega_{p}$. The solid lines are for the linear growth rates and the dashed lines for the frequencies. The cross marked line represents nonlinear oscillation frequencies.

has more peaks that are the combinations of two main peaks $f_{1}$ and $f_{2}$ [Fig. 3(c)]. The shape of return map (or Poincaré section) distorts from a circle [Fig. $3(\mathrm{~g})]$ and the system finally becomes chaotic as in Figs. 3(d) and 3(h).

Figure 3 was obtained from kinetic simulations by varying $\phi_{\mathrm{dc}}$ from 0.01 to 0.05 with $\alpha / \pi=2.96, \phi_{\mathrm{ac}}=0.02$. When $\phi_{\mathrm{dc}}<0.02$, the system shows period one oscillation with the external frequency as in Figs. 3(a) and 3(e). If the dc voltage is increased to the regime of $0.02<\phi_{\mathrm{dc}}<0.038$, the amplitude of the self-oscillation frequency increases to the level of that of the external frequency before the system state becomes quasiperiodic. If the dc voltage is increased over $\phi_{\mathrm{dc}}$ $=0.038$, the system becomes chaotic and finally enters the unstable state for $\phi_{\mathrm{dc}}>0.06$.

The self-oscillation frequency is more sensitive to the Pierce parameter than to the driving voltage. If the system is scanned by varying $\alpha$, two main frequencies become commensurate and mode-locked oscillations are found as in Fig. 4. The self-oscillation frequency is approximately (2.02.2) $\times 10^{6} \mathrm{~Hz}$ when the mode locking occurs while the external driving frequency is fixed at $1.2 \times 10^{6} \mathrm{~Hz}$ so the winding numbers are $3 / 5,4 / 7,5 / 9,6 / 11$ for the self-oscillation frequency $2.0 \times 10^{6}, 2.1 \times 10^{6}, 2.16 \times 10^{6}$, and $2.2 \times 10^{6} \mathrm{~Hz}$, respectively. This gives period $5,7,9,11$, and 14 mode-locked oscillations. The $14 P$ oscillations are the period doubled states from the $7 P$ oscillations as shown in Figs. 4(b) and 4(e).

There appears a mode locking of a different style. If $V_{\mathrm{ac}}$ is raised higher than $V_{\mathrm{dc}}$, the peak of the external frequency in the power spectrum grows so high compared to that of the self-oscillation frequency that the system shows a period one oscillation as in Fig. 4(f). If $V_{\text {ac }}$ is increased further, the system becomes unstable. There are also period doublings and chaotic regimes between the period one and the unstable state, which are hard to find because their regimes are very narrow.

Mode lockings are obtained by varying $\omega_{e}$ with a fixed $\alpha$. Mode-locked oscillations with various winding numbers

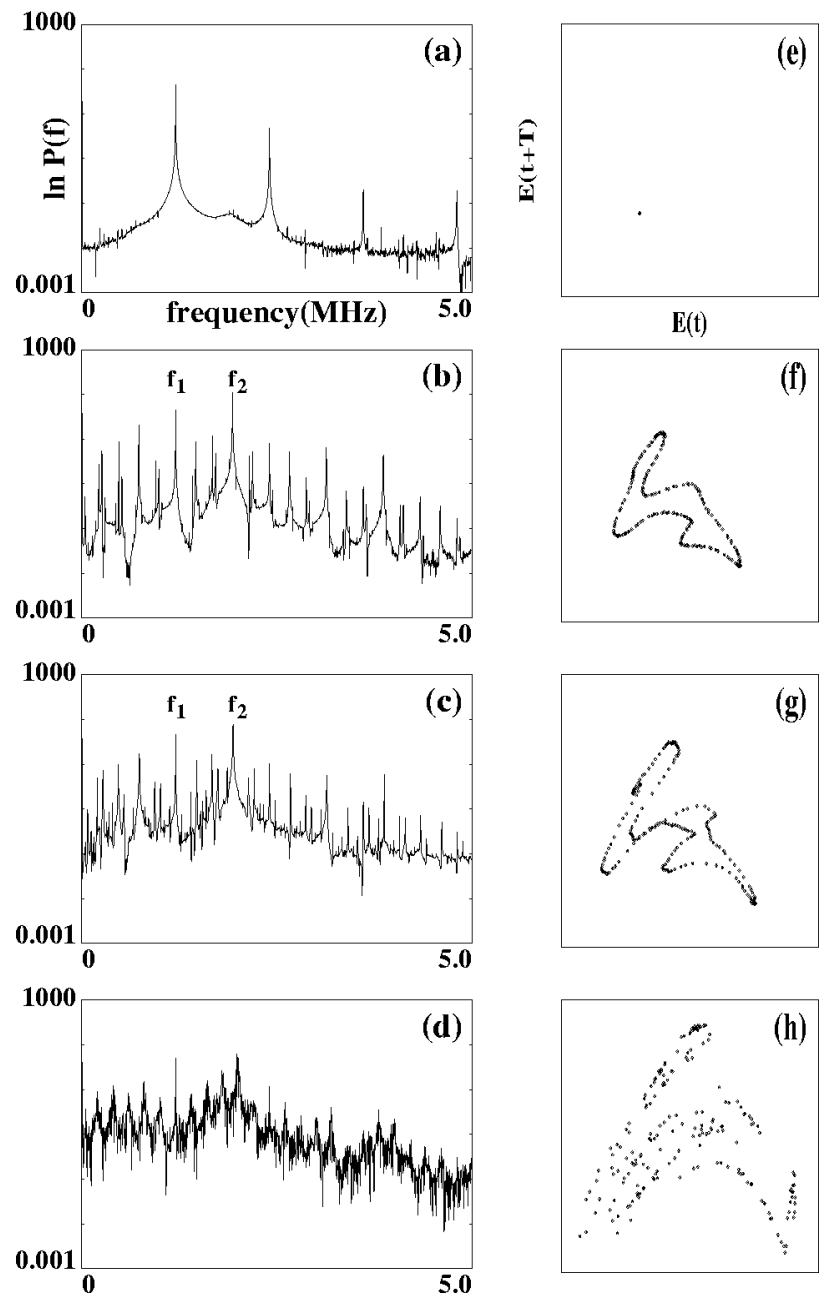

FIG. 3. The power spectra of QP signals and their return maps. $\phi_{\mathrm{ac}}, \alpha$, and the external frequency $\omega_{e}$ are fixed: $\phi_{\mathrm{ac}}=0.02, \alpha / \pi$ $=2.96, \omega_{e}=1.2 \times 10^{6} \mathrm{~Hz}$. The system becomes chaotic as $\phi_{\mathrm{dc}}$ is increased. (a) $1 P, \phi_{\mathrm{dc}}=0.01$. (b) $\mathrm{QP}, \phi_{\mathrm{dc}}=0.037$. (c) $\mathrm{QP}, \phi_{\mathrm{dc}}$ $=0.04$. (d) Chaos, $\phi_{\mathrm{dc}}=0.05$.

were found and mode suppression was observed near the resonance [28].

\section{Input parameter windows}

The previous studies are carried out by varying one parameter with the other parameters fixed at specified values. It will be useful for further study or design of similar systems to obtain the overall features of the system states in the parameter spaces. $\phi_{\mathrm{dc}}$ versus $\alpha / \pi$ diagrams with $\phi_{\mathrm{ac}}=0$ are plotted for various ion-electron ratio $\eta$ in Fig. 5(a) from kinetic simulations. This figure is nearly the same as our fluid-code result Fig. 1 of [22]. Both kinetic and fluid results are in good agreement except for the slight shifts of the boundary lines. When the system is in the regime of periodic or chaotic oscillations, there is no kinetic effect because the velocity distribution of the electrons in the beam is approximately the Dirac delta function. In the kinetic simulations the position of boundaries is somewhat influenced by the simulation cell size and the simulation particle number.

A period doubling can be obtained by increasing the dc voltage or decreasing the Pierce parameter. In the neighborhood of $\alpha / \pi=3$ and $\phi_{\mathrm{dc}}=0.1$, the period doubling occurs as 

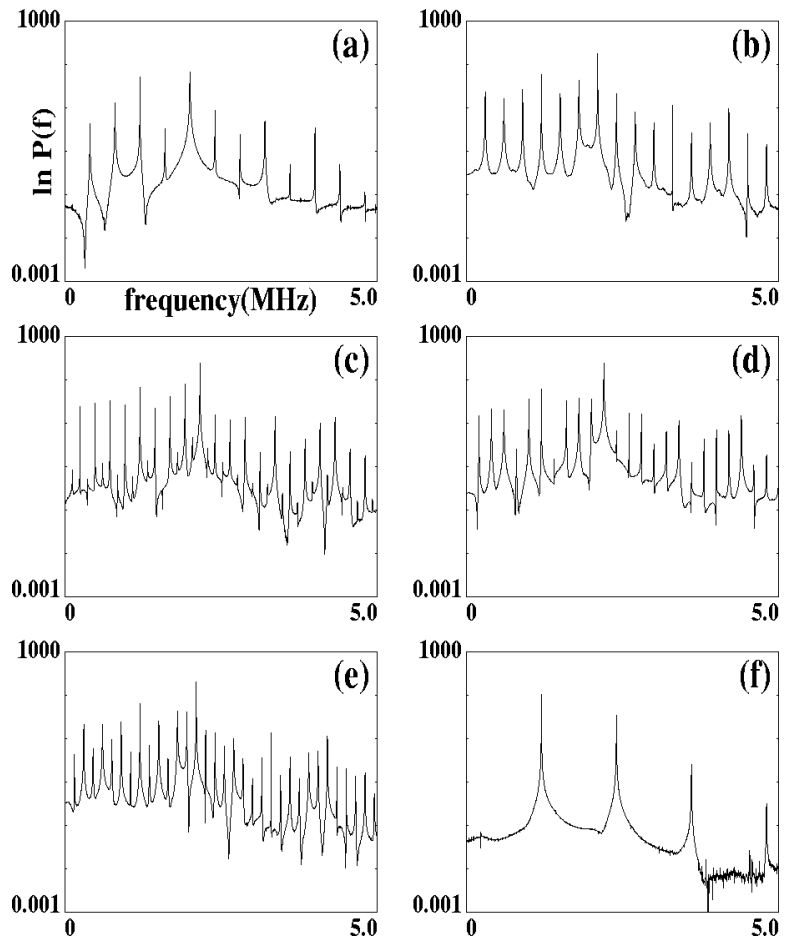

FIG. 4. The power spectra of mode-locked states. (a) $5 P, \alpha / \pi$ $=2.98, \phi_{\mathrm{dc}}=0.04, \phi_{\mathrm{ac}}=0.03$. (b) $7 P, \alpha / \pi=3, \phi_{\mathrm{dc}}=0.07, \phi_{\mathrm{ac}}$ $=0.03$. (c) $9 P, \alpha / \pi=3.02, \phi_{\mathrm{dc}}=0.1, \phi_{\mathrm{ac}}=0.03$. (d) $11 P, \alpha / \pi$ $=3.04, \phi_{\mathrm{dc}}=0.11, \phi_{\mathrm{ac}}=0.03$. (e) $14 P($ period doubled from $7 P)$, $\alpha / \pi=3, \phi_{\mathrm{dc}}=0.08, \phi_{\mathrm{ac}}=0.03$. (f) $1 P, \alpha / \pi=3, \phi_{\mathrm{dc}}=0.06, \phi_{\mathrm{ac}}$ $=0.08$.

$\alpha$ varies in both directions. When $\eta$ is changed, the boundary lines are shifted. Only the unstable-chaos boundaries are denoted in Fig. 5(a). Some intermittencies were also found but their regime is too narrow to be shown here.

If a small ac voltage is applied with a dc voltage, a wide area in the $\phi_{\mathrm{dc}}$ versus $\alpha / \pi$ parameter window is replaced by a QP regime as in Fig. 5(b). It is easily seen that QP is the main route to chaos. The period one oscillation in this case is somewhat different from that of the dc driven case. In the dc driven case, $1 P$ is self-activated by the internal instability but in the ac-dc driven case, the $1 \mathrm{P}$ oscillation is due to the external ac driving force. If the dc voltage is raised or the Pierce parameter is decreased, there also appears a selfactivated oscillation that interacts with the externally driven oscillation making QP. In Fig. 5(b), 1 P and QP correspond to the stable focus and $1 P$ of dc driven cases. The modelocked regime in the parameter window is too narrow to be shown here.

\section{COMMON DIAGNOSTIC MEASURE}

In the study of FEL chaos [16-18], a diagnostic $\mu$ was used to describe the nonlinear states. The nonlinear states are the complex function of the input paramters $S$ and $K$ and $D$ as shown in Fig. 12 of Ref. [17]. If the same feature is seen in the $\mu$ diagram, the system dependence on the input parameters becomes very weak compared with the dependence on $\mu$; namely, the system state is decided predominantly by $\mu$ as in Fig. 1 of Ref. [18]. It is a dimensionless parameter empirically defined as in [18]:
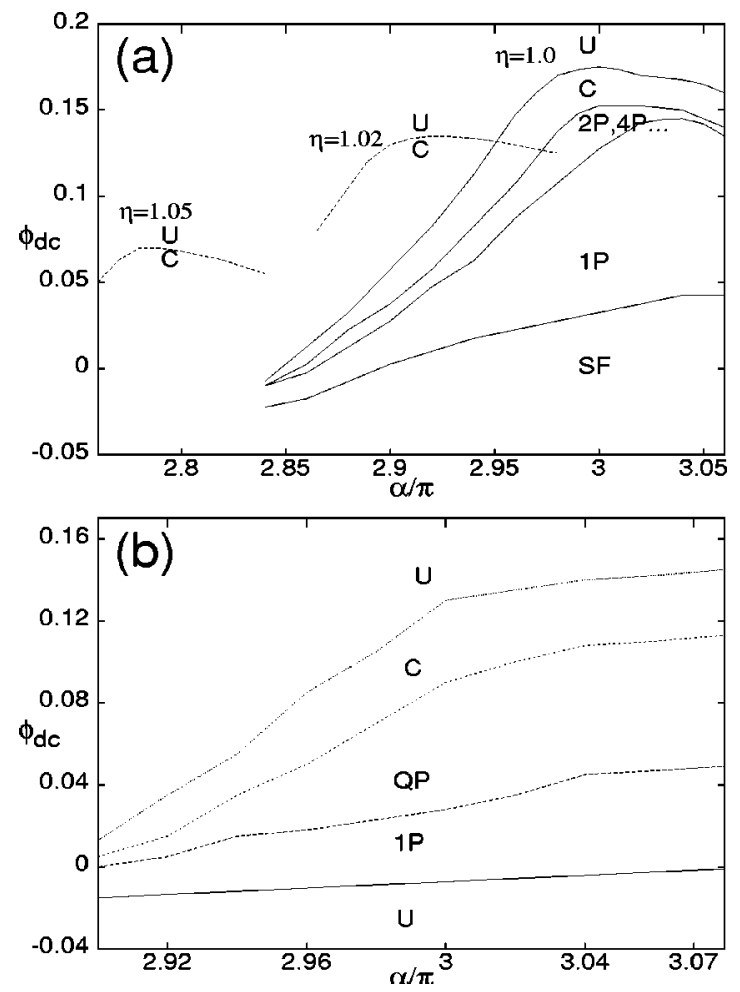

FIG. 5. $\phi_{\mathrm{dc}}$ vs $\alpha / \pi$ windows from kinetic simulations. (a) dc driven case. (b) ac and dc driven case for $\phi_{\mathrm{ac}}=0.01$.

$$
\mu=\frac{L_{s}}{L_{\mathrm{syn}}}=N_{w} \lambda\left[\frac{2 \pi c\left(c-u_{0}\right)}{\omega_{b} u_{0}}\right]^{-1},
$$

where $L_{s}$ is the interaction length between the electron beam and the electromagnetic wave and $L_{\text {syn }}$ is the slippage length, which is related to the velocity difference.

$\mu$ is defined in a similar manner to Eq. (11) in the plasma diode in [22]:

$$
\mu=\frac{L_{s}}{L_{\text {slip }}}=\frac{\pi u_{0}}{\omega_{p}}\left[\frac{2 \pi v_{\phi}\left(u_{0}-v_{\phi}\right)}{\omega_{b} u_{0}}\right]^{-1}=\frac{\omega_{b} / \omega_{r}}{2\left(1-v_{\phi} / u_{0}\right)},
$$

where $u_{0}$ is the initial velocity of the electron beam and $v_{\phi}$ is the phase velocity of the wave inside the diode. The bounce frequency $\omega_{b}$ is defined as $\sqrt{e k E / m}$ with wave number $k$ and maximum electric field $E$ at the cathode. $\mu$ contains the effects of two factors: the system length, which is related to the interaction length $L_{s}=\pi u_{0} / \omega_{p}$, and the field intensity, which is correlated with the bounce frequency. It is noted that the velocity difference (slippage) between the electron beam and the wave is also an important factor in determining the system's nonlinear state.

Figure 6 shows the $\mu$ diagrams from the kinetic simulations. Figure 6(a) is plotted with $\mu$ values calculated from data points in the regime of $2.84<\alpha / \pi<3.0$ and -0.02 $<\phi_{\mathrm{dc}}<0.18$ for the dc driven cases. For the ac and dc driven cases, $\mu$ values were calculated from data points in the regime of $2.96<\alpha / \pi<3.08,0.03<\phi_{\mathrm{dc}}<0.11$, and $0.01<\phi_{\mathrm{ac}}$ $<0.04$ to be plotted in terms of $\mu$ versus $\alpha / \pi, \mu$ versus $\phi_{\mathrm{dc}}$, and $\mu$ versus $\phi_{\text {ac }}$ in Figs. 6(b)-6(d). The system states depend much more strongly on $\mu$ than on the input parameters $\alpha, \phi_{\mathrm{dc}}, \phi_{\mathrm{ac}}$. The $\mu$ boundary of chaos and periodic oscilla- 

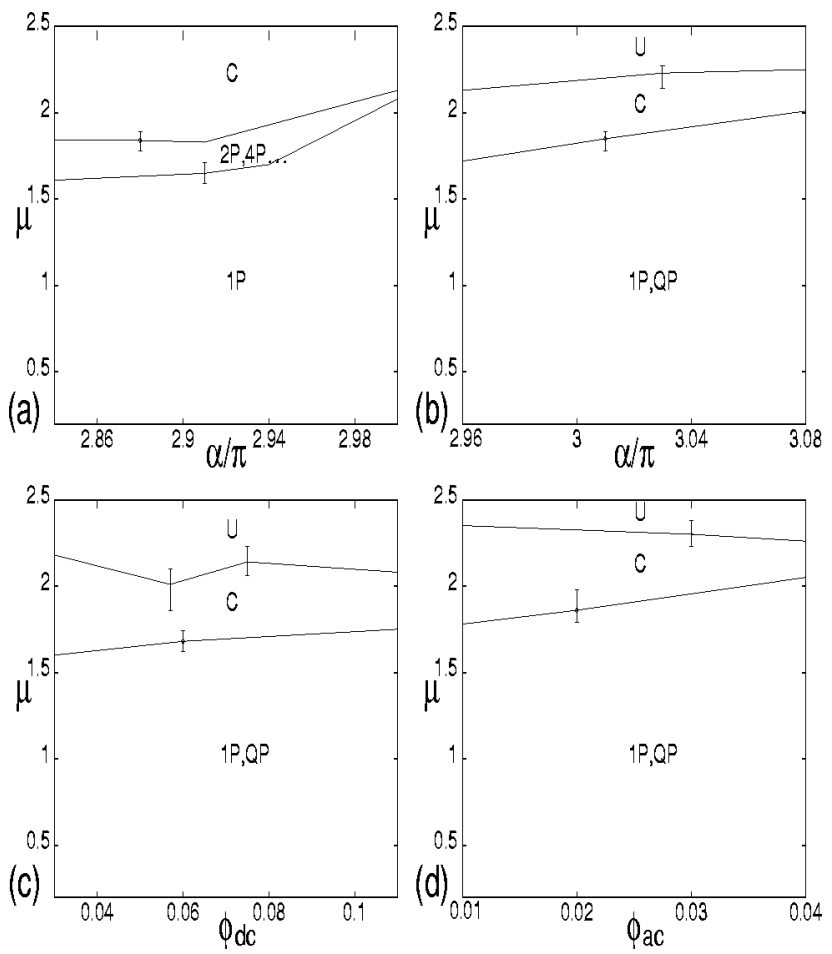

FIG. 6. The diagrams in terms of $\mu$ and input parameters. (a) $\mu$ vs $\alpha / \pi$ for dc driven case. (b) $\mu$ vs $\alpha / \pi$ for ac and dc driven case. (c) $\mu$ vs $\phi_{\mathrm{dc}}$ for ac and dc driven case. (d) $\mu$ vs $\phi_{\mathrm{ac}}$ for ac and dc driven case. The location of the boundaries varies within error bars as the input parameters are changed.

tion is about 1.7-2 regardless of the input parameters. In the FEL case, there are two regimes of chaos-the first is near $\mu \sim 1.5$ and the second is where $\mu>2$. The boundary of this second regime in FEL [18] is in good agreement with that of the plasma diode, despite that two systems are quite different. A larger value of $\mu$ was associated with more stochastic phenomena than a smaller $\mu$ case in the homogeneous beamplasma instabilities in an unbounded system [29]. For ac-dc driven cases shown in Figs. 6(b), 6(c), and 6(d), there are large QP regimes instead of $1 P$ in Fig. 6(a).

If $V_{\mathrm{dc}}$ is fixed small compared with $V_{\mathrm{ac}}$ and the system is driven raising ac voltage, quasiperiodic oscillation becomes a $1 P$ mode-locked oscillation and finally becomes chaotic as shown in Fig. 4(f). Therefore the ratio of $V_{\mathrm{ac}}$ to $V_{\mathrm{dc}}$ is a useful quantity to describe the system state. Figure 7 shows a parameter window of $\phi_{\mathrm{ac}}$ versus $\phi_{\mathrm{dc}}$ for $\alpha / \pi=3$. In the higher ac voltage region, the $1 P$ mode-locked state is dominant and the chaotic region is not observed. It is possible that a chaotic region still exists but was not found in the kinetic simulations due to the limitation in the numerical precision. In the lower ac voltage region, the QP to chaos route is dominant with mode lockings. The $\mu$ values of various states are shown in Fig. 7. The $\mu$ distributions of chaos and QP regimes $(1.75<\mu<2.5$ for chaos, $0.41<\mu<1.8$ for $\mathrm{QP})$ agree with Fig. 6 but the $\mu$ range of $1 P$ regime is very wide $(0.2<\mu<1.56)$. It is related to the mode locking. The $7 P$ $14 P$ mode-locked regime has $\mu$ spreading from 1.4 to 2.0 , which is comparable to that in the chaotic regime. As seen in Fig. 7, the $7 P$ mode-locked region is located close to the chaos region. The $1 P$ of large $\mu$ is a mode-locked $1 P$ by high ac voltage and that of small $\mu$ corresponds to the stable

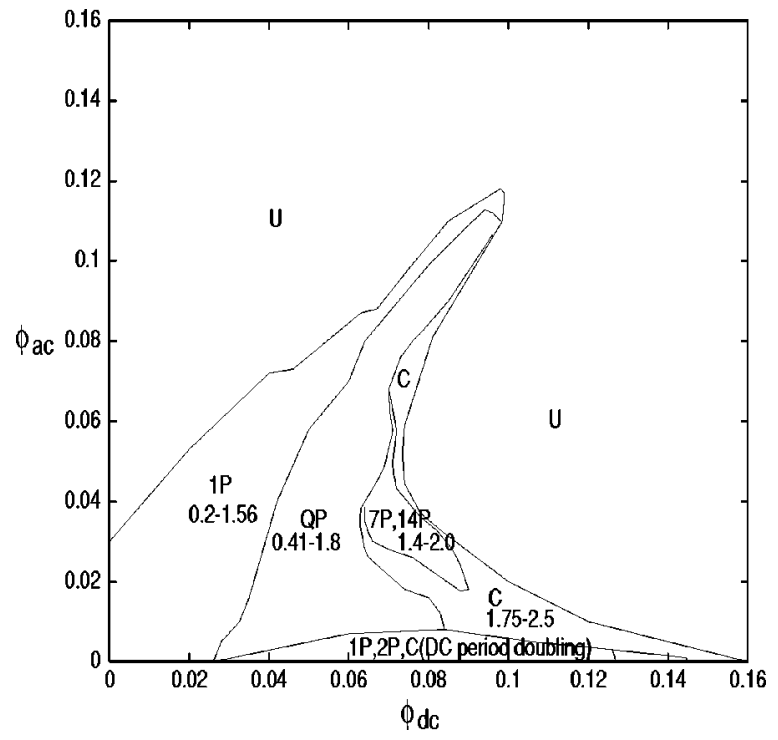

FIG. 7. Various oscillations in the $\phi_{\mathrm{ac}}$ vs $\phi_{\mathrm{dc}}$ parameter window. $\alpha / \pi$ is fixed at 3 .

focus of de driven system; this type of $1 P$ will be called "stable $1 P$ " to be distinguished from the mode-locked $1 P$. The mode-locked $1 P$ occurs in the strong self-oscillation regime, where the large amplitude of oscillation makes $\mu$ large by Eq. (12). The stable $1 P$ is driven only by an external ac voltage without a self-oscillation. The oscillation amplitude is so small that $\mu$ is small. It is the reason why the clear boundary between QP and $1 P$ is not obtained in the $\mu$ diagrams.

\section{SUMMARY}

Period doubling and quasiperiodicity were found in driven plasma-diodes. Period doubling is the main route to chaos in wholly dc driven cases [22] and also appears in ac driven cases. QP is not found in de driven systems [22] but is a dominant route when a small ac driving force exists. Mode lockings of $5 P, 7 P, 9 P, 11 P$, and $14 P$ are observed in some parameter regimes and $1 P$ mode lockings are also found when the system is driven by a high ac voltage. When the system is driven by a high ac voltage, QP disappears and the chaotic regime is too narrow to be observed. The parameter windows are obtained in terms of controllable input parameters.

A potentially universal diagnostic $\mu$ [22] in analogy with FEL chaos [18] is used for the driven plasma diode. dc and ac driven plasma diodes are fairly well described by $\mu$. The boundary between periodic oscillation and the chaos is approximately $\mu=1.7-2$. The simulations are carried out by two different codes (a particle code and a fluid code), both of which agree well.

\section{ACKNOWLEDGMENTS}

The present studies were supported (in part) by the BSRI Special Fund of POSTECH, the Basic Science Research Institute Program, Ministry of Education, Project No. 97-2439, and the PDP Center. 
[1] J.R. Pierce, J. Appl. Phys. 15, 721 (1944).

[2] Brendan B. Godfrey, Phys. Fluids 30, 1553 (1987).

[3] F. Greiner, T. Klinger, H. Klostermann, and A. Piel, Phys. Rev. Lett. 70, 3071 (1993).

[4] F. Greiner, T. Klinger, and A. Piel, Phys. Plasmas 2, 1810 (1995).

[5] T. Klinger, F. Greiner, A. Rohde, and A. Piel, Phys. Plasmas 2, 1822 (1995).

[6] C. Arnas. Capeau, G. Bachet, and F. Doveil, Phys. Plasmas 2, 4650 (1995).

[7] B. Albrecht, H. Deutsch, R.W. Leven, and C. Wilke, Phys. Scr. 47, 196 (1993).

[8] G.A. Held and Carson Jeffries, Phys. Rev. Lett. 56, 1183 (1986).

[9] C. Wilke, R.W. Leven, and H. Deutsch, Phys. Lett. A 136, 114 (1989).

[10] Ding Weixing, Huang Wei, Wang Xiaodong, and C.X. Yu, Phys. Rev. Lett. 70, 170 (1993).

[11] S.H. Fan, S.Z. Yang, J.H. Dai, S.B. Zheng, D.P. Yuan, and S.T. Tsai, Phys. Lett. A 164, 295 (1992).

[12] J. Qin, L. Wang, D.P. Yuan, P. Gao, and B.Z. Zhang, Phys. Rev. Lett. 63, 163 (1989).

[13] P.Y. Cheung, S. Donovan, and A.Y. Wong, Phys. Rev. Lett. 61, 1360 (1988).

[14] M. Bohm and S. Torven, Phys. Rev. Lett. 66, 604 (1991).
[15] M. Hörhager and S. Kuhn, Phys. Fluids B 2, 2741 (1990).

[16] S.J. Hahn and J.K. Lee, Phys. Lett. A 176, 339 (1993).

[17] S.J. Hahn and J.K. Lee, Phys. Rev. E 48, 2162 (1993).

[18] S.J. Hahn, J.K. Lee, E.H. Park, and T.H. Chung, Nucl. Instrum. Methods Phys. Res. A 341, 200 (1994).

[19] S.J. Hahn and J.K. Lee, Nucl. Instrum. Methods Phys. Res. A 331, 390 (1993).

[20] E.H. Park, J.K. Lee, and T.H. Chung, Nucl. Instrum. Methods Phys. Res. A 358, 448 (1995).

[21] X. Chen and P. Linsay, IEEE Trans. Plasma Sci. 24, 1005 (1996).

[22] H.J. Lee, J.K. Lee, M.S. Hur, and Y. Yang, Appl. Phys. Lett. 72, 1445 (1998).

[23] J.P. Verboncoeur, M.V. Alves, V. Vahedi, and C.K. Birdsall, J. Comput. Phys. 104, 321 (1993).

[24] William S. Lawson, Phys. Fluids B 1, 1483 (1989).

[25] V.M. Smirnov, Zh. Éksp. Teor. Fiz. 50, 1005 (1966) [Sov. Phys. JETP 23, 668 (1966)].

[26] M.J. Feigenbaum, J. Stat. Phys. 19, 25 (1978); 20, 669 (1979).

[27] S. Newhouse, D. Ruelle, and F. Takens, Commun. Math. Phys. 64, 35 (1978).

[28] A. Buragohain, Joyanti Chutia, and Y. Nakamura, Phys. Lett. A 163, 425 (1992).

[29] H.J. Lee, J.K. Lee, I.D. Bae, and M.S. Hur (unpublished). 